CLINICAL STUDY

\title{
Delayed pubertal onset and development in German children and adolescents with type 1 diabetes: cross-sectional analysis of recent data from the DPV diabetes documentation and quality management system
}

\author{
Tilman Rohrer, Eva Stierkorb, Sabine Heger ${ }^{1}$, Beate Karges ${ }^{2}$, Klemens Raile ${ }^{4}$, K Otfried Schwab ${ }^{5}$ and \\ Reinhard W Holl ${ }^{3}$ on behalf of the Diabetes-Patienten-Verlaufsdaten (DPV) Initiative \\ Pediatric Diabetes Unit, Department of Pediatric and Adolescent Medicine, Saarland University Hospital, Homburg/Saar, Germany, ${ }^{1}$ Pediatric Diabetes \\ Unit, Hospital for Children and Adolescents, University of Leipzig, Leipzig, Germany, ${ }^{2}$ Pediatric Diabetes Unit, Hospital for Children and Adolescents and \\ ${ }^{3}$ Department of Epidemiology, University of Ulm, Ulm, Germany, ${ }^{4}$ Pediatric Diabetes Unit, Hospital for Children and Adolescents, Charité, Berlin, \\ Germany and ${ }^{5}$ Pediatric Diabetes Unit, Hospital for Children and Adolescents, University of Freiburg, Freiburg, Germany
}

(Correspondence should be addressed to T Rohrer who is now at Department of Pediatrics and Neonatology, Saarland University Hospital, Kirrberger Str. 1, Geb. 9, 66421 Homburg/Saar, Germany; Email: kitroh@uniklinikum-saarland.de)

\begin{abstract}
Objective: To investigate the effect of type 1 diabetes on pubertal onset and development, and to identify factors potentially affecting puberty, including glycemic control, relative diabetes duration, body mass index standard delta score (BMI SDS), insulin dose, and intensity of insulin therapy.

Research design and methods: Initiated in 1990, the Diabetes-Patienten-Verlaufsdaten (DPV) is an ongoing, prospective longitudinal follow-up program to benchmark the quality of diabetes care provided to, predominantly, pediatric patients. Data collection for this non-interventional audit was carried out at 202 German diabetes treatment centers. Patient recruitment was done by referral, clinic/hospital ascertainment, or self-report. Data were analyzed for subcohorts of 1218-2409 boys and 579-2640 girls from a cohort of 24385 pediatric type 1 diabetic patients. Selection was based on ethnicity and availability of data on Tanner stage 2, or higher, of genital and pubic hair development (boys) or breast and pubic hair development, and menarche (girls).

Results: Boys showed significant $(P<0.05)$ delay (years) in mean ages at onset of genital development $(12.0( \pm 0.9)$ years $)$ and pubarche $(12.2( \pm 0.4)$ years $)$. In girls, mean ages at thelarche $(11.4( \pm 0.5)$ years), pubarche (11.5 ( \pm 0.1$)$ years), and menarche $(13.2( \pm 0.5)$ years) were significantly delayed compared with the general population. Sexual maturity (Tanner stage 5) was not delayed in either sex. Elevated glycohemoglobin and decreased BMI SDS were associated with significantly delayed pubertal onset, whereas relative diabetes duration and insulin dose were not.

Conclusions: Pubertal onset, but not sexual maturity, is delayed in children with type 1 diabetes. Delay increases with higher glycohemoglobin and lower BMI SDS.
\end{abstract}

European Journal of Endocrinology 157 647-653

\section{Introduction}

The currently available data indicate that pubertal onset and growth spurt are delayed in children and adolescents with type 1 diabetes mellitus (1). Literature reports on the effect of diabetes on age at menarche, however, have been inconsistent. Some, mostly retrospective, studies have reported delayed menarche in type 1 diabetes (2-4), whereas others found no difference compared with the general population (5-7). Factors such as age at onset of puberty or the quality of diabetes treatment appear to play important roles $(3,5,7,8)$.

After several conceptual changes since its inception in 1990, the DPV documentation and quality management system comprises three basic modules: a) the DPV software for prospective diabetes documentation; $b$ ) the benchmarking and quality control procedure (QC-DPV); and c) DPV-SCIENT, a cumulative diabetes research database (9). Using the DPV software, currently more than 200 participating centers regularly enter their data into the database on a semi-annual basis.

Based on the prospective longitudinal DPV database, this study undertook to compare with the data available for the general population the age at onset of puberty and pubertal development in a population-based cohort of German children and adolescents with type 1 diabetes, and to provide a cross-sectional analysis of the potential effects of glycemic control, body mass index (BMI), relative diabetes duration, insulin dose, and insulin therapy 
intensity on pubertal onset and development, including age at menarche.

\section{Research design and methods}

\section{Subjects}

Anonymous longitudinal data for a cohort of 24385 pediatric type 1 diabetic patients (12 756 boys and 11629 girls) aged 7.0 to $<17.0$ years (mean 13.9 years) with documented age of diabetes onset were selected for cross-sectional statistical analysis based on the following main inclusion criteria: a) age $<20$ years at last visit prior to January 1, 2006 and b) ethnicity (both parents born in Germany). Stages of pubertal development and sexual maturity were defined as Tanner stages of puberty $(10,11)$. Sexual maturity was defined as the attainment of Tanner stage 5 of genital (boys), breast (girls), and pubic hair (both sexes) development. Data on Tanner stages were available for a total of 13627 children and adolescents (6853 (50.3\%) boys and 6774 (49.7\%) girls). Data on Tanner stage 2 as a marker of pubertal onset were available for 5045 patients (2409 (47.7\%) boys and 2636 (52.3\%) girls). Datasets for both Tanner stage 2 and at least one independent variable were available for 4987 patients (2409 (48.3\%) boys and 2578 (51.7\%) girls). Menarche and diabetes onset data were available for 643 out of 11413 girls, of whom 579 had complete datasets for multivariate analysis. The patients selected for analysis of Tanner stage 2 and menarche were in the age ranges 7.0 to $<17.0$ and 8.0 to $<18.0$ years respectively. Patients with celiac disease were excluded.

\section{Data collection}

Data were obtained as of December 31, 2005, from the database of the DPV, the German diabetes documentation, and quality management system (9), to which 202 participating diabetes care centers (hospitals, clinics, and diabetes specialists in private practice) contributed data twice yearly. The data were verified, corrected at the original center if necessary, and entered into the database using the Visual FoxPro 9.0 (Microsoft, Redmond, WA, USA) derived DPV software for standardized prospective longitudinal documentation of diabetes in children, adolescents, and adults (12). Each center complied with local ethical and data management guidelines as reported previously (13). All data were collected during routine care. The responsible ethics committee was informed of the study and had no concerns.

\section{Dependent variables}

Age at onset of puberty The age for each Tanner stage of pubertal development was calculated as the examination date at which it was first observed minus the date of birth. Age of pubertal onset was defined as the attainment of Tanner stage 2 of genital (scrotal) development (boys), breast development (girls), or pubic hair growth (boys and girls), whichever occurred first.

Age at menarche This was calculated as the date of first menses reported at the first subsequent examination minus the date of birth.

For the purposes of the present analysis, all age data were rounded to the first decimal place.

Overall mean ages at Tanner stages 2-5 and mean age at menarche in the general population of eastern Germany served as reference data in the absence of standard values for Germany as a whole. The reference data were based on longitudinal multicenter data from a cohort initiated in the former German Democratic Republic in the mid-1980s as by Greil \& Kahl in 2005 (14).

\section{Independent variables}

HbA1C The quality of glycemic control was assessed by $\mathrm{HbA1c}$. $\mathrm{HbA}_{1 \mathrm{c}}$ levels were determined using the methodology established at each individual center. To adjust for differences among participating laboratories, the $\mathrm{HbA}_{1 \mathrm{c}}$ data were mathematically standardized according to the Diabetes Control and Complications Trial (DCCT) reference range of $4.05-6.05 \%\left(\mathrm{HbA}_{1 \mathrm{c}}\right.$-DCCT) (15).

Relative diabetes duration This was the proportion of a patient's life with type 1 diabetes, calculated as the duration of diabetes relative to chronological age at Tanner stage 2 or menarche.

BMI SDS The BMI $\left(\mathrm{kg} / \mathrm{m}^{2}\right)$ was calculated as a patient's body weight in kilograms divided by the square of his/her height in meters. To account for the non-normal distribution of the BMI in the population, BMI SD scores (BMI SDS) were calculated according to the LMS method (16) as adapted by Kromeyer-Hauschild et al. (17) for calculating standard percentile curves of BMI for children and adolescents in Germany. Normative BMI data for German children, adolescents, and adults used in the DPV software were based on KromeyerHauschild et al. (17) and Hebebrand et al. (18).

Insulin dose This was analyzed as daily units of insulin per kilogram body weight.

Intensity of insulin therapy This was represented by the mean number of daily insulin injection time points, with regimens ranging from one to seven daily injections. 


\section{Statistical analyses}

All statistical analyses were carried out using SAS software (SAS Version 9.1, SAS Institute, Cary, NC, USA) and the Statistical Package for Social Sciences (SPSS Base 13; SPSS Inc., Chicago, IL, USA). Values of $P<0.05$ were considered to indicate statistical significance in all analyses. Using the 'mixed' SAS procedure, stepwise multivariate linear regression analyses were performed for patients whose datasets were complete in respect of the independent variables of interest in order to identify variables influencing age at onset of puberty (Tanner stage 2) and age at menarche. Mean values were tested for significant difference using the $t$-test for unpaired samples. Differences between variances were tested using the F-test.

\section{Results}

Table 1 presents the mean value \pm s.D., median, and interquartile range (IQR) for age of pubertal onset (Tanner stage 2), as well as other clinical characteristics for 4987 children and adolescents $(<20$ years $)$ for whom Tanner stage 2 data were available from the German DPV database.

In addition, the mean age ( \pm s.D. $)$ at menarche observed in a subcohort of 643 girls was $13.22( \pm 1.31)$ years, and the median age was 13.00 years (IQR, 12.30-14.00 years). Diabetes onset occurred at a mean age ( \pm s.D.) of $8.37( \pm 3.27)$ years in this subcohort. The respective means $( \pm$ s.D. $)$ for diabetes duration at menarche and relative diabetes duration (proportion of life with type 1 diabetes) at menarche were $2.05( \pm 2.96)$ and 0.28 $( \pm 0.25)$ respectively. The BMI SDS was $0.20( \pm 0.85)$. The overall median $\mathrm{HbA}_{1 \mathrm{c}}$ and $\mathrm{HbA}_{1 \mathrm{c}}$-DCCT values were $7.80( \pm 1.75)$ and $7.94( \pm 1.71) \%$ respectively. The mean insulin dose in the menarche subcohort was 0.84 $( \pm 0.25) \mathrm{U} / \mathrm{kg}$ per day, and the mean number of daily insulin injections was $3.42( \pm 1.13)$.

As shown in Table 2, analysis of the data for our study cohort revealed highly significant delay in the onset of puberty as defined by Tanner stage 2 of genital (scrotal) development (G2) or pubic hair growth (PH2) in diabetic boys and breast development (B2) or pubic hair growth (PH2) in diabetic girls. Significant delay was also observed for the subsequent stages of pubertal development up to G4 and PH4 in boys and B 3 and PH4 in girls. Sexual maturity as evidenced by Tanner stages $\mathrm{G} 5$ and $\mathrm{PH} 5$ in diabetic boys and $\mathrm{B} 5$ and $\mathrm{PH} 5$ in diabetic girls, however, occurred at statistically non-significantly earlier ages than in the reference population, while the B4 ages for diabetic and healthy girls were virtually identical. Comparisons of mean ages for Tanner stages were based on reference data for the general population of eastern Germany derived from a multicenter study of a cohort initiated in the mid-1980s (14).

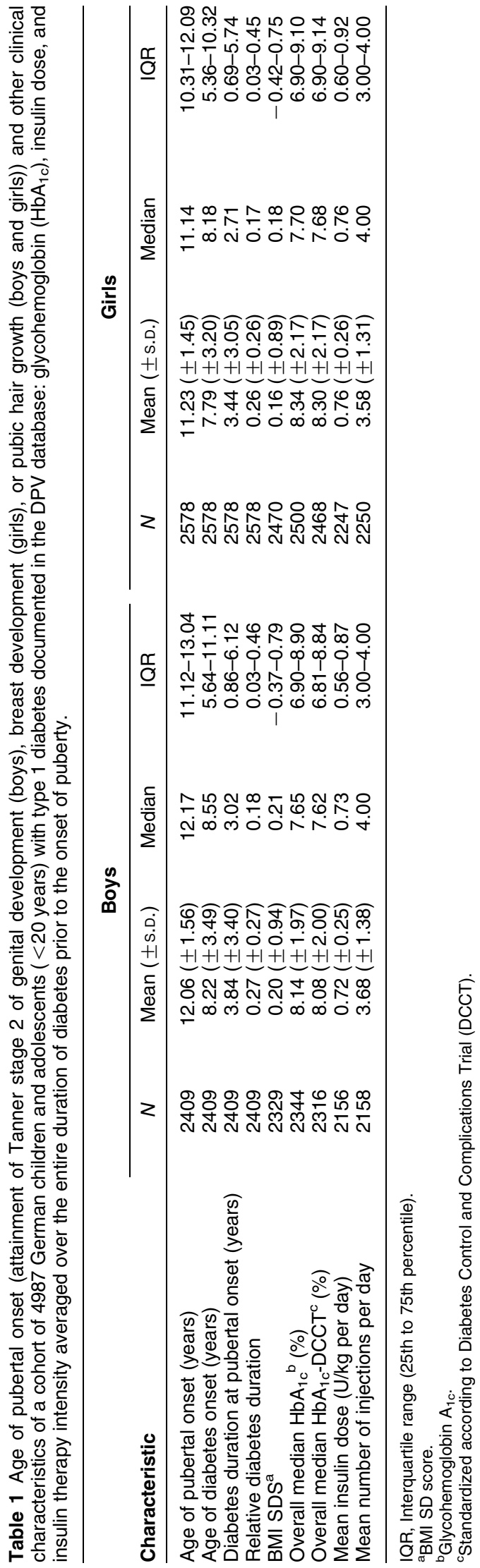

www.eje-online.org 


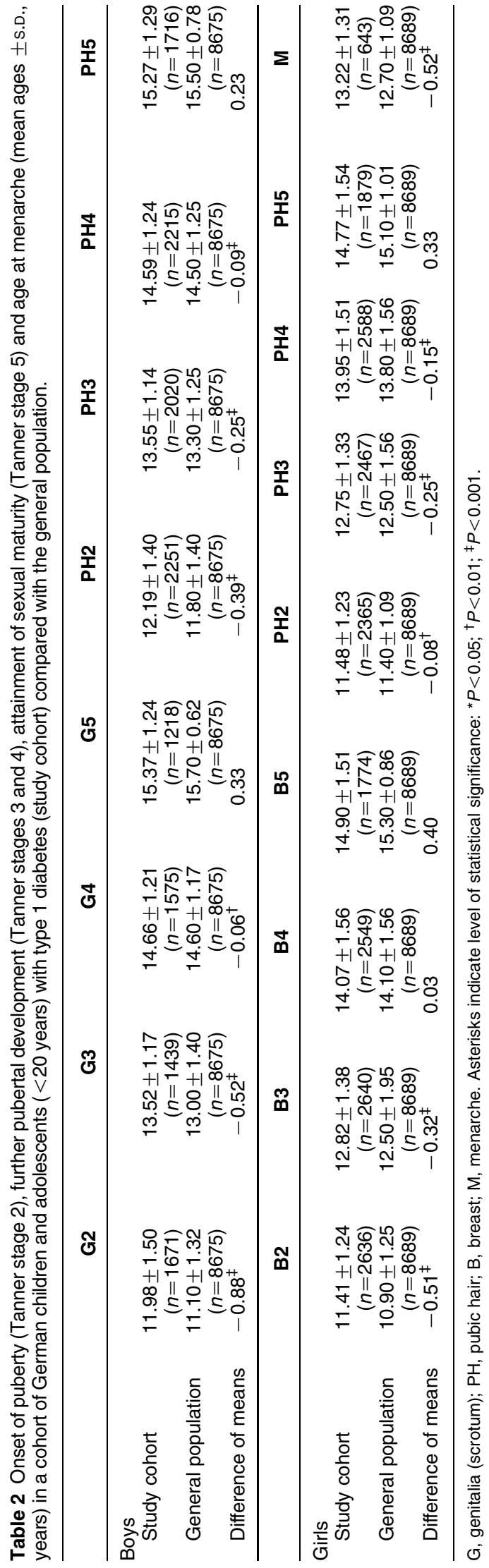

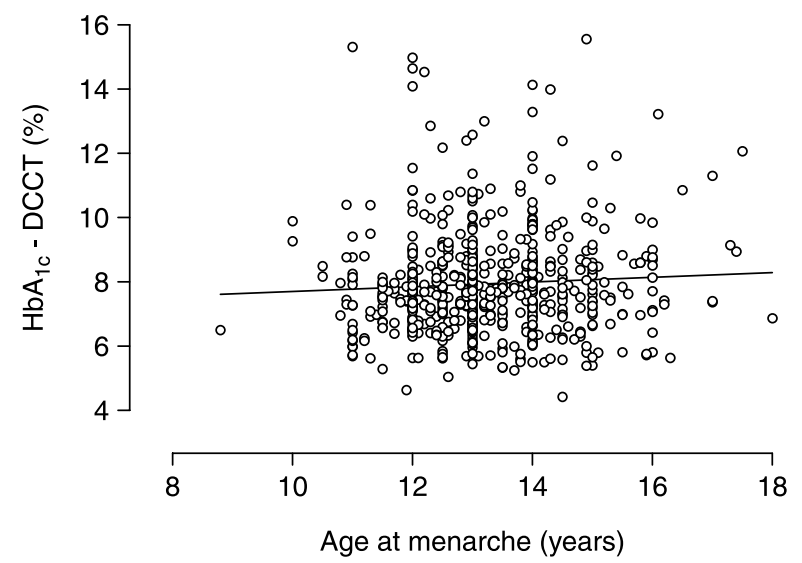

Figure 1 Association of age at menarche and $\mathrm{HbA}_{1 \mathrm{c}}-\mathrm{DCCT}(\%)$ in girls up to the age of $<18$ years at menarche. There was a trend for age at menarche to increase with $\mathrm{HbA}_{1 \mathrm{c}}$ (Spearman's $r=0.06, P<0.05$ ).

Mean age at menarche in our study cohort was highly significantly delayed by 0.52 years, compared with the reference data. In addition, linear regression analysis of the raw data (not shown; $N=615$ ) revealed that a $1 \%$ increase in $\mathrm{HbA}_{1 \mathrm{c}}$-DCCT was associated with a delay in menarche by 0.07 years $(\sim 4$ weeks, Fig. 1$)$. Also, girls who at the time of menarche had had type 1 diabetes for half their lives (relative diabetes duration 0.5 ) experienced their first menses at an average of 0.47 years later than those whose diabetes onset coincided with first menses (relative diabetes duration 0.0), again according to the linear regression estimate based on the raw data for 615 girls (Fig. 2).

Table 3 summarizes the statistically significant associations revealed by multivariate analysis of age of pubertal onset (Tanner stage 2) and age at menarche in our study cohort as the dependent variables versus a number of potentially influencing independent variables. $\mathrm{HbA}_{1 \mathrm{c}^{-}}$ DCCT and BMI SDS were significantly associated with delay and advancement of pubertal onset respectively, in a

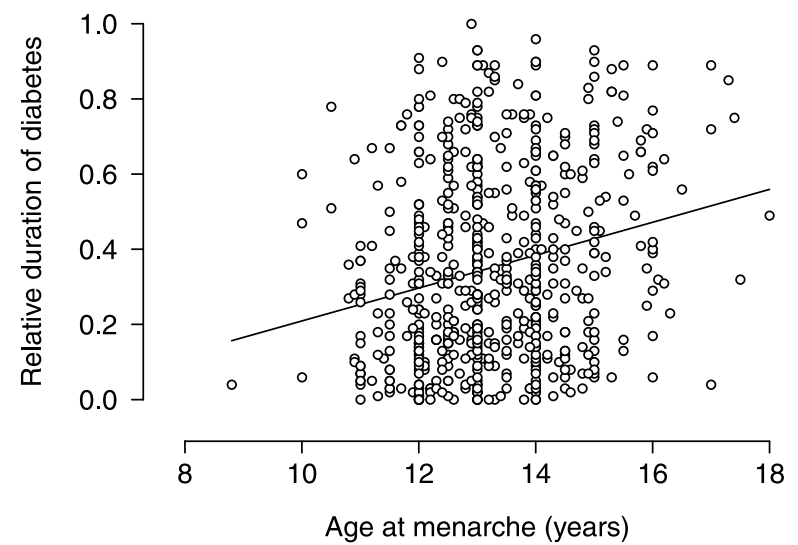

Figure 2 Association of age at menarche and relative diabetes duration in girls up to the age of $<18$ years at menarche. There was a trend for age at menarche to increase with relative diabetes duration (Spearman's $r=0.96, P<0.0001$ ). 
Table 3 Statistically significant multivariate associations with age of pubertal onset and age at menarche (years) in a cohort of German children and adolescents ( $<20$ years) with type 1 diabetes mellitus.

\begin{tabular}{|c|c|c|c|c|}
\hline Multivariate analysis for & $N$ & $\beta$ value & S.E.M. $(\beta)$ & $P$ value \\
\hline \multicolumn{5}{|l|}{ Age of pubertal onset: all patients } \\
\hline Estimated intercept $( \pm$ S.E.M. $): 10.85( \pm 0.22) ; P<0.0001$ & 4231 & & & \\
\hline \multicolumn{5}{|l|}{ Significantly associated independent variables } \\
\hline $\mathrm{HbA}_{1 \mathrm{c}}$ & 4231 & 0.10 & 0.01 & $<0.0001$ \\
\hline BMI SDS & 4231 & -0.38 & 0.02 & $<0.0001$ \\
\hline Sex: male & 4231 & 0.90 & 0.04 & $<0.0001$ \\
\hline \multicolumn{5}{|l|}{ Age of pubertal onset: boys alone } \\
\hline Estimated intercept ( \pm S.E.M.): $11.69( \pm 0.30) ; P<0.0001$ & 2073 & & & \\
\hline \multicolumn{5}{|l|}{ Significantly associated independent variables } \\
\hline $\mathrm{HbA}_{1 \mathrm{c}}$ & 2073 & 0.10 & 0.02 & $<0.0001$ \\
\hline BMI SDS & 2073 & -0.34 & 0.03 & $<0.0001$ \\
\hline \multicolumn{5}{|l|}{ Age of pubertal onset: girls alone } \\
\hline Estimated intercept $( \pm$ s.E.M. $): 11.00( \pm 0.32) ; P<0.0001$ & 2158 & & & \\
\hline \multicolumn{5}{|l|}{ Significantly associated independent variables } \\
\hline $\mathrm{HbA}_{1 \mathrm{c}}$ & 2158 & 0.10 & 0.02 & $<0.0001$ \\
\hline BMI SDS & 2158 & -0.44 & 0.03 & $<0.0001$ \\
\hline \multicolumn{5}{|l|}{ Age at menarche } \\
\hline Estimated intercept ( \pm s.E.M.): $13.00( \pm 0.73) ; P<0.0001$ & 579 & & & \\
\hline \multicolumn{5}{|l|}{ Significantly associated independent variables } \\
\hline $\mathrm{HbA}_{1 \mathrm{c}}$ & 579 & 0.06 & 0.03 & 0.0436 \\
\hline BMI SDS & 579 & -0.60 & 0.06 & $<0.0001$ \\
\hline Relative diabetes duration & 579 & 0.96 & 0.21 & $<0.0001$ \\
\hline Daily insulin dose & 579 & -0.61 & 0.22 & 0.0064 \\
\hline
\end{tabular}

similar and consistent manner for all patients, and for boys and girls alone. The advancement of pubertal onset associated with increasing BMI SDS was greater in the girls-alone analysis $(\beta=-0.44)$ than in the all-patients analysis $(\beta=-0.38)$, the effect of this variable being least pronounced in boys alone $(\beta=-0.34)$. Male sex was a significantly associated variable in the all-patients analysis. No significant associations were obtained for individual numbers (one to seven) of daily insulin injections with the $t$-test.

Multivariate analysis of 579 complete menarche datasets revealed statistically significant effects on age at menarche for $\mathrm{HbA}_{1 \mathrm{c}}$-DCCT, BMI SDS, relative diabetes duration, and insulin dose, as shown in Table 3. The associations for $\mathrm{HbA}_{1 \mathrm{c}}$-DCCT and relative diabetes duration were positive, indicating that delay in menarche increased with either variable, while the associations for BMI SDS and insulin dose were negative, indicating an advancement of age at menarche. Again, no significant associations were obtained for individual numbers (one to seven) of daily insulin injections with the $t$-test.

\section{Discussion}

To our knowledge, this is the first study in a large cohort of German children and adolescents with type 1 diabetes to analyze the effect the disease may have on the onset of puberty and pubertal development, including age at menarche, and to investigate potential associations between markers of pubertal onset and development and factors that may affect the process of sexual maturation, such as nutritional status, chronic disease, or environmental factors (19-22).

As a chronic disease also occurring in childhood, type 1 diabetes is a factor potentially affecting the onset of male and female puberty and pubertal development, including age at menarche. Our analysis of current DPV data showed type 1 diabetes to be associated with significant mean delays in pubertal onset by 0.88 and 0.39 years for the male G2 and PH2 Tanner stages and by 0.51 and 0.08 years for the female B2 and $\mathrm{PH} 2$ Tanner stages respectively, compared with healthy boys and girls in the German general population. Our analysis further indicated that the differences between the study cohort and the general population decreased for Tanner stages 3-5. This suggests that diabetic children catch up to healthy children as pubertal development progresses and ultimately reach sexual maturity at a normal age, as evidenced by - statistically non-significantly - earlier attainment of Tanner stage 5 of genital (boys), breast (girls), and pubic hair (both sexes) development.

Interestingly, however, menarche, a mid-pubertal event, was found to occur with a 0.52-year delay at a mean age of 13.22 years in type 1 diabetic girls, although pubertal development at that age had already reached the normal female Tanner stages B3-B4 and PH3-PH4. Possible causes of the observed delay in menarcheal age in type 1 diabetic girls may lie at the hypothalamic-pituitary level. Clinically, female patients with type 1 diabetes may show oligomenorrhea and amenorrhea $(2,8,23)$. There have also been reports of decreased luteinizing hormone (LH) levels, suggesting impairment of the hypothalamic-pituitary axis (24). 
Furthermore, since delayed menarche is a potential risk factor for irregular menstrual cycles and decreased bone mineral density possibly leading to later subfertility and osteoporosis (3), evidence confirming menarcheal delay may have clinical implications for improving the treatment of diabetes to help prevent such subsequent complications.

$\mathrm{Hb}_{1 \mathrm{c}}$ was found in our study cohort to correlate significantly with the age of pubertal onset in boys and girls together, as well as in boys alone and girls alone, and with age at menarche, the latter confirming previous findings of a study from the United States (3). One possible explanation for this correlation is that type 1 diabetes is associated with markedly increased serum levels of glycosylated products (25), indicating that such advanced glycation end products, e.g., various proteins, may act to suppress activation of the LH-releasing hormone (LHRH) pulse generator during puberty, resulting in pubertal delay in general and delayed menarche in particular. Another explanation might be that elevated serum $\mathrm{HbA}_{1 \mathrm{c}}$ levels indicate an overall chronic lack of insulin. It is well known that insulin not only signals satiety in the hypothalamus but is also involved in the regulation of reproductive function as demonstrated in vitro and in vivo (26-28). Knockout mice lacking the neuronal insulin receptor experience central hypogonadism, reduced spermatogenesis, and impaired maturation of ovarian follicles (26). Furthermore, intact mice respond to artificially increased serum insulin levels with increases in $\mathrm{LH}$ levels due to a hypothalamic insulin effect (27). There is evidence that insulin might regulate LHRH neuronal function directly via insulin receptors expressed on LHRH neurons (28). Disruption of normal LHRH neuronal function due to lack of insulin might be present in type 1 diabetic children, resulting in delayed onset of puberty.

Another notable finding in our present study was that age of pubertal onset was significantly associated with BMI SDS in boys and girls together, boys alone, and girls alone. Menarche was significantly inversely associated with BMI SDS and tended to occur earlier in type 1 diabetic girls with a high BMI SDS than in those with a low BMI SDS. This is in agreement with the established fact that nutritional status plays an important role in the onset and progression of female puberty. While premature puberty can be triggered by an increased BMI $(22,29)$, malnutrition leads to a delay in the onset of puberty and menarche (21). Moreover, delayed puberty, and menarche in particular, has been reported in girls who participate in competitive sports (19).

Age at menarche was also found to be significantly associated with relative type 1 diabetes duration in our study. The longer the premenarcheal period with type 1 diabetes, the greater was the delay in the onset of first menses. This observation lends support to the hypothesis that, in the presence of diabetes mellitus, antibodies to ovarian cells impair ovarian function (30). Thus, the earlier the onset of type 1 diabetes occurs during the prepubertal period, the greater is the likelihood that such antibodies could have already formed and resulted in ovarian functional impairment. However, the present study did not experimentally investigate this hypothesis.

A delay in menarche and/or irregularity of the menstrual cycle could also be indicative of ovarian dysregulation. As ovarian insulin receptors play an important role in ovarian function, increased $\mathrm{HbA}_{1 \mathrm{c}}$ levels due to the lack of tightly regulated insulin levels may affect ovarian maturation and function, and hence pubertal development in type 1 diabetes (31). Based on this explanation, insulin dose would be expected to have a normalizing effect on age of pubertal onset and age at menarche. Our study confirmed this effect for age at menarche but not for pubertal onset. Girls treated with a higher insulin dose in our study cohort experienced their first menses earlier, whereas those on a lower dose had their first menses later. However, this finding was also not unexpected since insulin dosage depends on body weight, among other factors.

To summarize, analysis of the DPV data for our study cohort revealed significant delay in pubertal onset and age at menarche, though ultimately sexual maturity, i.e., Tanner stage 5, was attained at a normal age. We conclude that type 1 diabetes can cause delay in the onset of male and female puberty and menarche, which increases with glycohemoglobin levels or, conversely, decreases with improved glycemic control. Inversely, decreases in BMI SDS are associated with delayed pubertal onset and menarche. Regarding menarche, we additionally conclude that the longer the prepubertal duration of type 1 diabetes, i.e., the earlier diabetes onset occurs, the more likely menarche is to be delayed. In addition, menarcheal age is associated with insulin dose but not with the number of daily injections. In conclusion, it is of great clinical importance to improve glycemic control, as the most readily modifiable factor, to reduce delay in male and female pubertal onset and menarche in type 1 diabetic children.

\section{Acknowledgements}

The DPV program was supported by the German Federal Ministry of Health, the German Diabetes Society (DDG), the Dr Bürger-Büsing Foundation, the German Research Foundation (DFG), the National Action Forum on Diabetes Mellitus (NAFDM), the German Medical Association (Bundesärztekammer), and Novo Nordisk Pharma GmbH, Germany. The authors are grateful to Profs Ludwig Gortner and Norbert Graf for helpful discussions, and wish to acknowledge all patients, investigators, and staff who participated in the DPV initiative. A full listing of the participating centers has recently been published elsewhere (32). 


\section{Disclosure of potential duality or conflicts of interest}

T Rohrer, E Stierkorb, S Heger, B Karges, K Raile, K O Schwab and R W Holl have nothing to declare.

\section{References}

1 Malone JI. Growth and sexual maturation in children with insulindependent diabetes mellitus. Current Opinion in Pediatrics 19935 494-498.

2 Burkart W, Fischer-Guntenhoner E, Standl E \& Schneider HP. Menarche, menstrual cycle and fertility in diabetic patients. Geburtshilfe und Frauenheilkunde 198949 149-154.

3 Danielson KK, Palta M, Allen C \& D'Alessio DJ. The association of increased total glycosylated hemoglobin levels with delayed age at menarche in young women with type 1 diabetes. Journal of Clinical Endocrinology and Metabolism 200590 6466-6471.

4 Tattersall RB \& Pyke DA. Growth in diabetic children. Studies in identical twins. Lancet 19732 1105-1109.

5 Ahmed ML, Connors MH, Drayer NM, Jones JS \& Dunger DB. Pubertal growth in IDDM is determined by $\mathrm{HbA}_{1 \mathrm{c}}$ levels, sex, and bone age. Diabetes Care 199821 831-835.

6 Salerno M, Argenziano A, Di Maio S, Gasparini N, Formicola S, De Filippo G \& Tenore A. Pubertal growth, sexual maturation, and final height in children with IDDM. Effects of age at onset and metabolic control. Diabetes Care 199720 721-724.

7 Schriock EA, Winter RJ \& Traisman HS. Diabetes mellitus and its effects on menarche. Journal of Adolescent Health Care 19845 101-104.

8 Yeshaya A, Orvieto R, Dicker D, Karp M \& Ben-Rafael Z. Menstrual characteristics of women suffering from insulin-dependent diabetes mellitus. International Journal of Fertility and Menopausal Studies $199540269-273$.

9 Grabert M, Schweiggert F \& Holl RW. A framework for diabetes documentation and quality management in Germany: 10 years of experience with DPV. Computer Methods and Programs in Biomedicine 200269 115-121.

10 Marshall WA \& Tanner JM. Variations in pattern of pubertal changes in girls. Archives of Disease in Childhood $1969 \mathbf{4 4}$ 291-303.

11 Marshall WA \& Tanner JM. Variations in pattern of pubertal changes in boys. Archives of Disease in Childhood 197045 13-23.

12 Holl RW \& Grabert M. The quality circle: how to improve the outcome of paediatric diabetes care. Hormone Research 200257 (Suppl 1) 105-109.

13 Reinehr T, Schober E, Wiegand S, Thon A \& Holl R. Beta-cell autoantibodies in children with type 2 diabetes mellitus: subgroup or misclassification? Archives of Disease in Childhood 200691 $473-477$.

14 Greil H \& Kahl H. Assessment of developmental age: crosssectional analysis of secondary sexual characteristics. Anthropologischer Anzeiger 200563 63-75.

15 The Diabetes Control and Complications Trial Research Group. The effect of intensive treatment of diabetes on the development and progression of long-term complications in insulin-dependent diabetes mellitus. New England Journal of Medicine $1993 \mathbf{3 2 9}$ 977-986.

16 Cole TJ \& Green PJ. Smoothing reference centile curves: the LMS method and penalized likelihood. Statistics in Medicine 199211 $1305-1319$.

17 Kromeyer-Hauschild K, Wabitsch M, Kunze D, Geller F, Geiß HC, Hesse V, von Hippel A, Jaeger U, Johnsen D, Korte W, Menner K, Müller G, Müller JM, Niemann-Pilatus A, Remer T, Schaefer F, Wittchen HU, Zabransky S, Zellner K, Ziegler A \& Hebebrand J.
Perzentile für den Body-mass-Index für das Kindes- und Jugendalter unter Heranziehung verschiedener deutscher Stichproben. Monatsschrift für Kinderheilkunde 2001149 807-818.

18 Hebebrand J, Heseker H, Himmelmann GW, Schäfer H \& Remschmidt H. Altersperzentilen für den Body-Mass-Index aus Daten der Nationalen Verzehrstudie einschließlich einer Übersicht zu relevanten Einflußfaktoren. Aktuelle Ernährungsmedizin 1994 $19259-265$.

19 Georgopoulos N, Markou K, Theodoropoulou A, Paraskevopoulou P, Varaki L, Kazantzi Z, Leglise M \& Vagenakis AG. Growth and pubertal development in elite female rhythmic gymnasts. Journal of Clinical Endocrinology and Metabolism $1999 \mathbf{8 4} 4525-4530$.

20 Kelly SD, Howe CJ, Hendler JP \& Lipman TH. Disordered eating behaviors in youth with type 1 diabetes. Diabetes Educator 2005 31 572-583.

21 Kulin HE, Bwibo N, Mutie D \& Santner SJ. The effect of chronic childhood malnutrition on pubertal growth and development. American Journal of Clinical Nutrition 198236 527-536.

22 Stark O, Peckham CS \& Moynihan C. Weight and age at menarche. Archives of Disease in Childhood 198964 383-387.

23 Kromeyer-Hauschild K, Zellner K, Jaeger U \& Hoyer H. Prevalence of overweight and obesity among school children in Jena (Germany). International Journal of Obesity and Related Metabolic Disorders 199923 1143-1150.

24 Griffin ML, South SA, Yankov VI, Booth RA, Jr, Asplin CM, Veldhuis JD \& Evans WS. Insulin-dependent diabetes mellitus and menstrual dysfunction. Annals of Medicine 199426 331-340.

25 Berg TJ, Dahl-Jorgensen K, Torjesen PA \& Hanssen KF. Increased serum levels of advanced glycation end products (AGEs) in children and adolescents with IDDM. Diabetes Care 199720 $1006-1008$

26 Bruning JC, Gautam D, Burks DJ, Gillette J, Schubert M, Orban PC, Klein R, Krone W, Muller-Wieland D \& Kahn CR. Role of brain insulin receptor in control of body weight and reproduction. Science $20002892122-2125$.

27 Burcelin R, Thorens B, Glauser M, Gaillard RC \& Pralong FP. Gonadotropin-releasing hormone secretion from hypothalamic neurons: stimulation by insulin and potentiation by leptin. Endocrinology $2003 \mathbf{1 4 4} 4484-4491$.

28 Salvi R, Castillo E, Voirol MJ, Glauser M, Rey JP, Gaillard RC, Vollenweider P \& Pralong FP. Gonadotropin-releasing hormoneexpressing neurons immortalized conditionally are activated by insulin: implication of the mitogen-activated protein kinase pathway. Endocrinology 2006147 816-826.

29 Wattigney WA, Srinivasan SR, Chen W, Greenlund KJ \& Berenson GS. Secular trend of earlier onset of menarche with increasing obesity in black and white girls: the Bogalusa Heart Study. Ethnicity and Disease 19999 181-189.

30 Snajderova M, Martinek J. Horejsi J. Novakova D, Lebl J \& Kolouskova S. Premenarchal and postmenarchal girls with insulin-dependent diabetes mellitus: ovarian and other organspecific autoantibodies, menstrual cycle. Journal of Pediatric and Adolescent Gynecology 199912 209-214.

31 Poretsky L, Cataldo NA, Rosenwaks Z \& Giudice LC. The insulinrelated ovarian regulatory system in health and disease. Endocrine Reviews 199920 535-582.

32 Schwab KO, Doerfer J, Hecker W, Grulich-Henn J, Wiemann D, Kordonouri O, Beyer P \& Holl RW. Spectrum and prevalence of atherogenic risk factors in 27358 children, adolescents, and young adults with type 1 diabetes: cross-sectional data from the German diabetes documentation and quality management system (DPV). Diabetes Care 200629 218-225.

Received 3 August 2007

Accepted 3 September 2007 